\title{
Examination of the mechanisms of osteolysis in patients with metastatic breast cancer
}

\author{
MATEYA TRINKAUS ${ }^{1}$, WEI SEONG OOI ${ }^{2}$, ETHAN AMIR ${ }^{1}$, \\ SNEZANA POPOVIC ${ }^{3}$, MARION KALINA ${ }^{4}$, HARIETTE KAHN ${ }^{2}$, \\ GURMIT SINGH $^{4}$, MARY CORONA GAINFORD ${ }^{5}$ and MARK CLEMONS ${ }^{1}$ \\ ${ }^{1}$ Princess Margaret Hospital; ${ }^{2}$ Toronto Sunnybrook Regional Cancer Centre, \\ Toronto; ${ }^{3}$ McMaster University; ${ }^{4}$ Juravinski Cancer Centre, Hamilton, \\ ON, Canada; ${ }^{5}$ University of Sydney, Sydney, NSW, Australia
}

Received March 26, 2008; Accepted May 15, 2008

DOI: 10.3892/or_00000335

\begin{abstract}
The benefits of bisphosphonates (BPs) in reducing skeletal-related events (SREs) in patients with bone metastases has mainly been attributed to their potent osteoclast inhibiting effect. However, despite the use of modern systemic anticancer therapy including potent BPs, many patients with bone metastases continue to have SREs. An improved understanding of the fundamental mechanisms of bone destruction allows for further development of appropriate targeted treatments. In this study, archival paraffin-embedded bone metastases specimens from patients with metastatic breast cancer were examined for the presence of osteoclasts, expression of the receptor activator of nuclear factor $\kappa \mathrm{B}$ (RANK), RANK Ligand (RANKL), osteoprotegerin (OPG) and vascular endothelial growth factor (VEGF). Histological specimens were available for primary breast cancer, lymph node metastases, normal breast and normal bone tissues for comparison. Bone metastasis specimens were available for 20 BP naïve patients and two BP-treated patients. Osteoclasts were significantly increased in the bone metastases of the BP naïve group compared to normal bone. No osteoclasts were detected in the BP-treated group. RANKL was predominantly expressed in osteoblasts and in the stromal elements of metastatic tissue. Conversely, RANK was present in osteoclasts of bone metastases and normal bone, as well as in tumor cells of metastatic lymph nodes and bone metastases. VEGF was strongly expressed in the control bone and bone metastases regardless of BP treatment. In summary, osteoclasts may not be the singular obligatory factor for osteolysis in bone metastases. An increased expression of RANKL in stromal
\end{abstract}

Correspondence to: Dr Mark Clemons, Princess Margaret Hospital, 610 University Ave, Toronto, ON M5G 2M9, Canada

E-mail: mark.clemons@uhn.on.ca

Key words: bone metastases, breast cancer, osteolysis, receptor activator of nuclear factor $\mathrm{\kappa B}$ ligand, vascular endothelial growth factor tissue surrounding bone metastases, RANK in osteoclasts and VEGF may serve as future targeted therapies possibly in conjunction with bisphosphonates. The mechanisms for osteoclast expression and the expression of RANKL, RANK, OPG and VEGF merit further prospective analysis, particularly in the context of BP treatment and progressive bone metastases.

\section{Introduction}

Breast cancer continues to be the most commonly diagnosed cancer in women in the developed world (1). Bone metastases are the most common site of metastatic recurrence (2) and are a major cause of morbidity for patients. Complications resulting from bone metastases include pain, reduced mobility and a reduced quality of life. In addition, patients are at a considerable risk of, so-called skeletal related events (SREs) such as the requirement for surgery and/or radiotherapy, pathological fractures, spinal cord compression and hypercalcaemia (3). Characteristically, patients with bone as the predominant site of metastatic spread tend to have a protracted disease course when compared to those patients with visceral metastases (4). Furthermore, patients with the disease confined to the skeleton have been found to be most likely to develop skeletal-related complications and hence derive the most benefit from treatment with bone-specific therapies (5).

Bone resorption is dependent on a cytokine known as the receptor activator of nuclear factor $\kappa \mathrm{B}$ ligand (RANKL), a tumor necrosis factor (TNF) family member that is essential for osteoclast formation, activity and survival in normal and pathological states of bone remodeling (6-8). The catabolic effects of RANKL are prevented by osteoprotegerin (OPG), a TNF receptor family member that binds RANKL and thereby prevents the activation of its single cognate receptor, RANK $(9,10)$. Osteoclast activity is likely to depend, at least in part, on the relative balance of RANKL and OPG. Studies on animal models show that the administration of RANKL leads to rapid skeletal changes of increased bone turnover, increased cortical porosity, weakened cancellous microarchitecture and reductions in bone volume, density and strength (11-13). 
Similar findings were observed in OPG knockout mice, where increased bone turnover was seen and associated with cortical porosity and reductions in cortical and cancellous bone volume and density (14-16).

Cancer cells have been shown to regulate OPG and RANKL pathways in a variety of ways. The net effect, however, appears to be an increase in the RANKL:OPG ratio in a manner that favors bone resorption. In breast cancer, in vitro studies have demonstrated that metastatic deposits can directly resorb bone, however, an increase in osteoclastic activity is considered to be the predominant mechanism for bone destruction (17). Unlike other cancers, breast cancer cells do not typically express RANKL directly (18-20), they can however, upregulate RANKL expression by osteoblasts $(17,18)$ and bone marrow stromal cells $(17,19,20)$. Furthermore, they can downregulate OPG production by osteoblasts and bone marrow stromal cells (18). The mechanism underlying this aberrant regulation is likely to be the secretion of a variety of cytokines such as Interleukin-1 (IL-1), parathyroid related peptide (PTHrP) and $\mathrm{TNF} \alpha(21)$.

Bisphosphonates (BPs) are synthetic analogues of naturally occurring pyrophosphate compounds. They are able to inhibit both the formation and dissolution of calcium phosphate crystals in bone, thus reducing bone turnover (22). However, in the setting of metastatic bone disease, the most important clinical effect is the inhibition of bone resorption. This is thought to be mediated principally by cellular mechanisms, including the inhibition of osteoclast recruitment and adhesion. The life span of osteoclasts is reduced, their activity is diminished and there appears to be modulation of the osteoclast-osteoblast interrelation (22).

Although BPs are associated with a reduction and delay in SREs (23), in clinical practice, many patients continue to have both SREs and progression of their bone metastases despite extensive BP use (24). The mechanism underlying this progression is unknown. Data from animal models have shown that even when tumor burden is evident in bone, osteoclasts are not necessarily present (25). The direct cause of continued bone destruction in the absence of identifiable osteoclasts is an area of active study.

In view of the majority of the above data being derived from animal models and in order to examine this area further in the in vivo setting, this study sought to determine the differences in osteoblast and osteoclast numbers as well as the differential expression of RANKL, RANK, OPG and Vascular endothelial growth factor (VEGF) in a variety of normal and metastatic tissue from a series of patients with metastatic breast cancer.

\section{Materials and methods}

A retrospective review of patients diagnosed with bone metastases from breast cancer between January 1998 and January 2005 was carried out at two centers in Ontario, Canada (Sunnybrook Health Sciences Center in Toronto and The Juravinski Cancer Centre in Hamilton). Patients who had undergone bone biopsies either as part of orthopedic procedures for management of the complication of bone metastases or bone biopsies to diagnose suspected bone metastases were identified from pathology records and electronic patient records. Relevant paraffin-embedded blocks of tissue of these patients were collected for evaluation. These samples included primary breast tumors, nodal metastases and bone metastases. For control purposes, a random sample of healthy breast and bone tissue biopsies was also examined. Immunohistochemical analysis was carried out on all these samples as well as their surrounding non-malignant stromal tissue if available.

Serial, 5- $\mu \mathrm{m}$ thick paraffin sections were deparaffinized, rehydrated through a graded ethanol series and rinsed in $50 \mathrm{mM}$ Tris-buffered saline with $0.1 \%$ Tween-80 (TBST). Heat induced epitope retrieval (HIER) was performed for $30 \mathrm{~min}$ at $95^{\circ} \mathrm{C}$ in citrate buffer, $\mathrm{pH}$ 6.0. After rinsing with TBST, the sections were incubated with $5 \%$ normal goat serum for $30 \mathrm{~min}$ at room temperature (RT). Primary antibody incubations were then carried out at RT for $1 \mathrm{~h}$, followed by a $30^{\circ} \mathrm{C}$-min incubation in either goat anti-rabbit or goat anti-mouse biotinylated secondary antibody (Vector Laboratories, Burlingame, CA) diluted at 1:500 in TBST pH 7.4. The endogenous peroxidase activity was then blocked with $0.3 \%$ $\mathrm{H}_{2} \mathrm{O}_{2}$ in TBST for $10 \mathrm{~min}$. Sections were then rinsed in TBST and incubated for $30 \mathrm{~min}$ with a Vectastain Elite ABC kit (Vector Laboratories) and developed in Nova Red Peroxidase substrate (Vector Laboratories). The sections were counterstained with Gill no.3 heamatoxylin solution prior to mounting.

Specimens of bony tissue also needed to undergo rapid decalcification by washing specimens thoroughly in running tap water for 2-3 min to remove residual formaldehyde. RDO rapid decalcifying fluid (Apex Engineering Products, Plainfield, IL) was then used by placing a cassetted specimen into freshly filtered decalcification fluid. The volume of decalcification fluid to tissue was maintained at a ratio of 20:1. Tissue was left in the decalcification fluid for 30-60 min, intermittently checking tissue for flexibility. The tissue was then transferred to a running tap water wash receptacle and washed for a minimum of $1 \mathrm{~h}$. The washed specimens were then placed in $10 \%$ neutral buffered formalin prior to processing.

Samples were tested for RANKL, RANK and OPG. A smaller subgroup was also tested for VEGF. Rabbit anti-RANK polyclonal antibody (AB1861 Chemicon International, Temecula, CA) was used at 1:50 dilution. Rabbit anti-RANKL polyclonal antibody (AB1862 Chemicon International) was used at 1:1000 dilution. Mouse anti-human OPG/TNFRSF11B monoclonal antibody (R\&D Systems, Minneapolis, MN) was used at 1:50 dilution. Rabbit anti-VEGF polyclonal antibody (A-20: sc-152 Santa Cruz Biotechnology Inc., CA) was used at a dilution of 1:20 overnight at $4^{\circ} \mathrm{C}$. Furthermore, in order to identify osteoclasts within biopsy material, Tartrateresistant acid phosphatase (TRAP) staining was performed using NCL-TRAP, clone 26E5 (Novocastra Ltd., Newcastle Upon Tyne, UK), at a dilution of 1:50. Histomorphometric variables such as osteoclast number were calculated following the American Society for Bone and Mineral Research (ASBMR) Histomorphometry Nomenclature Committee recommendations (26).

All bone immunohistochemistry and histomorphometry were compared to 20 control specimens, which consisted of bone unaffected by metastases. 


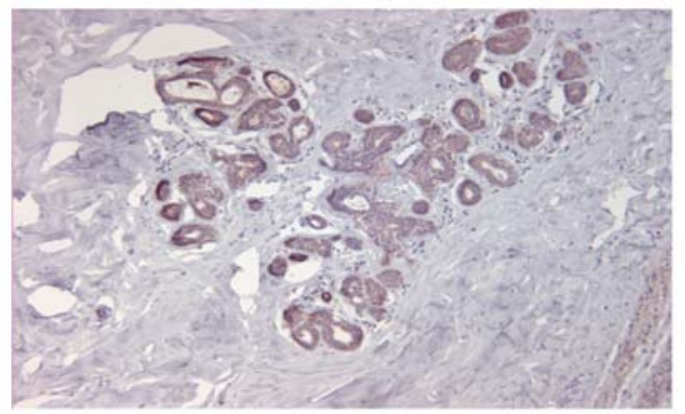

Normal Breast

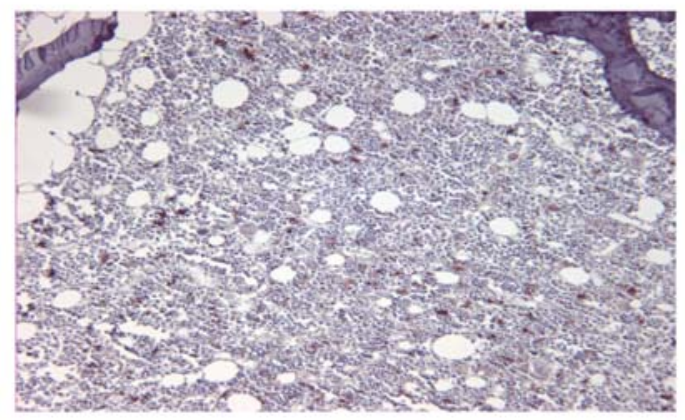

Normal Bone Marrow

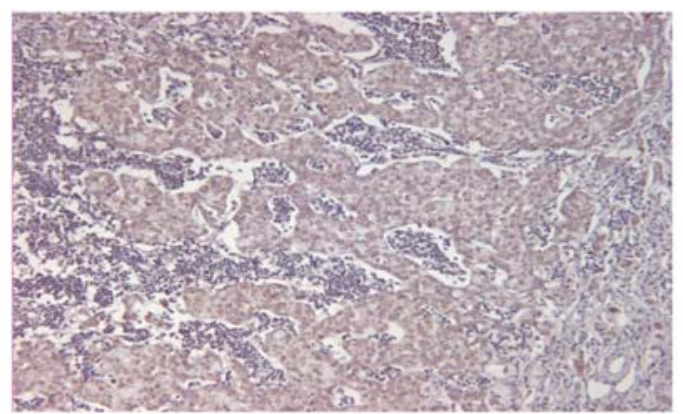

Involved Lymph Node

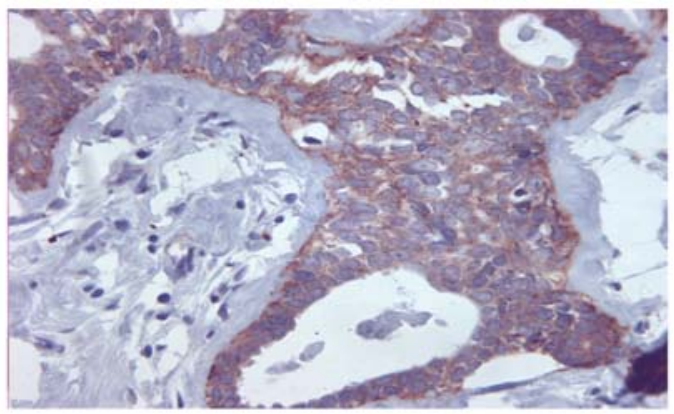

\section{Normal Breast}

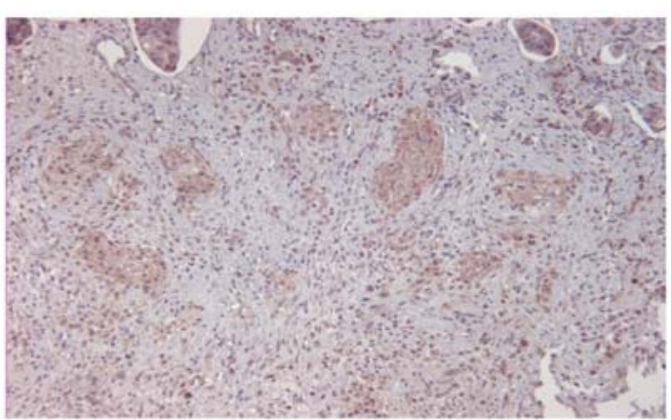

Bone Metastasis

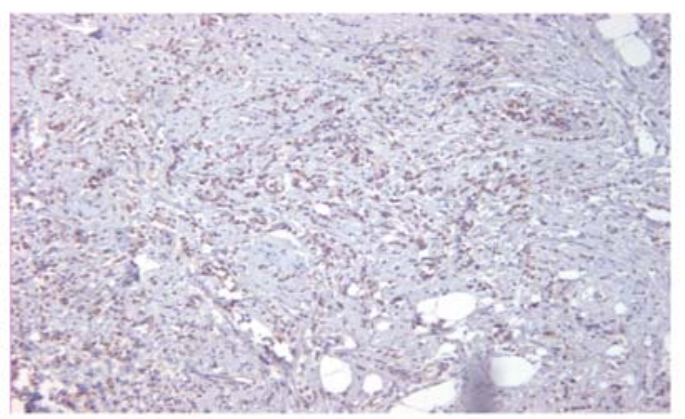

Primary Breast Cancer

Figure 1. Immunostaining of VEGF in normal breast, normal bone marrow, bone metastases, lymph node metastases and primary breast cancer specimen.

\section{Results}

Twenty-two patients with previously biopsied bone metastases from breast cancer were identified. Patients had a mean age of 54.9 years (range 39-79) and consisted of 20 patients who were BP naïve and 2 patients who were actively being treated with BPs (Table I). The majority of samples of bone metastases were obtained during surgery for pathological fractures or spinal cord compression.

In the 20 control bone specimens, the number of osteoclasts per unit of bone surface was $0.1^{+/-0} .04(/ \mathrm{mm})$. Among the 20 metastatic, BP naïve bone samples, an increase in number of osteoclasts per bone surface was seen $\left[1.9^{+/-3} .1(/ \mathrm{mm})\right.$ $(\mathrm{p}<0.05)]$. Notably, there were no osteoclasts detected in the two BP-treated metastatic bone specimens.

Immunohistochemical positivity for RANK was seen in breast cancer tumor cells in both bone and nodal metastases, although it was absent in the primary breast cancer tumor as well as in osteoclasts and normal bone stroma. RANKL was
Table I. Patient demographics.

\begin{tabular}{lcc}
\hline & BP naïve & BP-treated $^{\mathrm{a}}$ \\
\hline $\mathrm{N}$ & 20 & 2 \\
Mean age & $56(39$ to 79$)$ & 44 (42 to 46) \\
No. of fractures & 11 & 1 \\
No. of non-fractures & 5 & 1 \\
No. of occult fractures & 4 & 0 \\
\hline
\end{tabular}

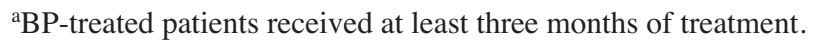

present in metastatic bone stroma and in osteoblasts, although not seen in any of the tumor cells or in the stroma of normal bone (Tables II and III). Unfortunately, OPG data could not be reported, as the data obtained was ambiguous, possibly due 
Table II. RANK/RANKL immunostaining in metastatic (BP naïve and BP-treated) and normal bone.

\begin{tabular}{|c|c|c|c|c|c|c|c|c|c|c|c|}
\hline & \multicolumn{4}{|c|}{ Metastatic bone (BP naïve) $(n=20)$} & \multicolumn{4}{|c|}{ Metastatic bone (BP-treated) $(n=2)$} & \multicolumn{3}{|c|}{ Control bone $(n=4)$} \\
\hline & TC & $\mathrm{SC}$ & $\mathrm{OC}$ & OB & $\mathrm{TC}$ & $\mathrm{SC}$ & $\mathrm{OC}$ & OB & $\mathrm{SC}$ & $\mathrm{OC}$ & OB \\
\hline RANK & $10 / 20$ & $0 / 20$ & $13 / 20$ & $2 / 20$ & $1 / 2$ & $0 / 2$ & $0 / 2$ & $0 / 2$ & $0 / 4$ & $4 / 4$ & $0 / 4$ \\
\hline RANKL & $0 / 20$ & $17 / 20$ & $1 / 20$ & $20 / 20$ & $0 / 2$ & $2 / 2$ & $0 / 2$ & $2 / 2$ & $0 / 4$ & $0 / 4$ & $4 / 4$ \\
\hline
\end{tabular}

Number of cases with the cell type positively stained. TC, tumor cells; SC, stromal cells; OC, osteoclasts and OB, osteoblasts.

Table III. RANK/RANKL immunostaining of primary breast carcinoma, metastatic lymph node and normal breast tissue.

\begin{tabular}{|c|c|c|c|c|c|c|}
\hline & \multicolumn{2}{|c|}{$\begin{array}{l}\text { Primary BCA } \\
\qquad(\mathrm{n}=4)\end{array}$} & \multicolumn{2}{|c|}{$\begin{array}{l}\text { Metastatic LN } \\
\quad(n=4)\end{array}$} & \multicolumn{2}{|c|}{$\begin{array}{l}\text { Normal breast } \\
\qquad(\mathrm{n}=4)\end{array}$} \\
\hline & $\mathrm{TC}$ & $\mathrm{SC}$ & $\mathrm{TC}$ & $\mathrm{SC}$ & BEPC & $\mathrm{SC}$ \\
\hline RANK & $0 / 4$ & $1 / 4$ & $3 / 4$ & $0 / 4$ & $0 / 4$ & $0 / 4$ \\
\hline RANKL & $0 / 4$ & $4 / 4$ & $0 / 4$ & $4 / 4$ & $0 / 4$ & $4 / 4$ \\
\hline
\end{tabular}

Number of cases with the cell type positively stained. BEPC, breast epithelial cells; BCA, breast carcinoma; LN, lymph node; SC, stromal cells and TC, tumor cells.

to cross reactivity of the antibody with carbonic anhydrase, as reported previously (27).

Four BP naïve bone specimens and the two BP-treated bone specimens were immunostained for VEGF expression. As shown in Fig. 1, tumor cells in each of the specimens were strongly positive for immunolocalization for VEGF. In normal tissue, VEGF staining was seen in endothelial cells and in normal bone marrow stroma.

\section{Discussion}

Bone remains the most common site of metastasis from breast cancer and is associated with significant skeletal complications. Studies by the British surgeon Stephen Paget over 100 years ago showed that certain cancer cells had a predilection to metastasize to bone and he proposed that the bone microenvironment provided a fertile 'soil' for the localization and growth of particular types of cancer cells (28). A century later, data have shown that products released from resorbing bone provided a chemotactic stimulus for tumor cell dissemination to bone (29). These observations, coupled with the ability of tumor cells to stimulate osteoclastic bone resorption (25), provided a powerful rationale for exploring bone turnover suppression as a therapeutic strategy for bone metastasis.

Randomized trials in breast cancer patients have shown that bisphosphonate therapy in combination with either endocrine therapy or chemotherapy can significantly delay the onset of SREs and also reduce their frequency (25,30-34). Moreover, in vitro studies have demonstrated that even though an increase in osteoclastic activity is considered to be the predominant mechanism for bone destruction, breast cancer deposits can directly resorb bone (17). As only osteoclast- mediated bone resorption can be inhibited by bisphosphonates (35), it would not be expected that all SREs could be prevented by bisphosphonates.

Studies 30 years ago hypothesized the existence of an 'osteoclast stimulating factor' and 'osteoclast activating factor' $(25,36)$, though it was not until the past decade that bone resorption was shown to be dependent on a group of regulatory cytokines, including dominant roles for OPG and RANKL (6-8). The presence of tumor cells in bone is thought to increase the RANKL:OPG ratio in a manner that favors bone resorption. The functional consequences of these regulatory influences have not been exhaustively studied in the in vivo setting. This study, albeit with a small number of subjects has explored these recently described pathways of osteolysis in metastatic breast cancer, specifically, it has attempted to assess the role of RANKL, OPG and VEGF pathways in patients with bone metastases from breast cancer.

RANKL was consistently detected in the stromal cells of normal breast tissue, primary breast cancers, metastatic lymph nodes and metastatic bone specimens in this study. It was also found in osteoblasts in the two normal and metastatic bone specimens. Importantly, it was not found within the stroma of any of the normal bone control specimens, nor was it present in primary or disseminated tumor cells. Conversely, the presence of RANK was identified in tumor cells in bone and in nodal metastases, though notably was not present in primary breast tumor cells. These findings are in contradiction with previous studies, which had detected the presence of RANKL in breast cancer tumor cells (37). However, the findings of this study are consistent with the results of a recent study, which evaluated the presence of RANKL mRNA expression in two human prostate cancer cell lines and found that these were absent in the primary tumor (38). Further supporting evidence comes from recent animal data (39), showing that osteolysis can occur after an injection of metastatic bone cells from prostate cancer into a tibia model despite treatment with a neutralizing antibody to human RANKL. This suggests that RANKL from the host's surrounding bone stroma (rather than from tumor cells) or other undetermined factors are upregulated to induce osteolysis.

Overall, the localized expression of RANKL by osteoblasts and by stroma surrounding metastatic tissue suggests an intimate relationship with RANK expressing tissue such as certain disseminated breast cancer cells. These findings can possibly be explained by the 'seed and soil' theory, which describes bone microenvironment as a fertile 'soil' for the localization of cancer cells (40). Furthermore, it would appear that normal bone resorption not only attracts tumor cells, but 
also stimulates their growth, while tumor cells promote osteoclast formation and activity, the result of which is more bone resorption that further attracts and stimulates cancer cells, the so called 'vicious circle' theory.

Evidence shows that RANKL alone cannot induce bone resorption and that other cytokines, such as the colony stimulating factor, must also be present for osteolysis to occur $(9,20)$. Recently, VEGF has been shown to support peripheral blood mononuclear cell differentiation into osteoclast-like cells, which were able to resorb substrate while in the presence of RANKL (41). It is possible that these cells provide the appropriate cytokine 'soup' necessary to induce osteolysis. Clearly, more studies are needed to explore this interaction further.

As documented previously, technical difficulties prevented any meaningful data being derived from the staining of tissue for OPG. This was predominantly due to the lack of reliable commercial antibodies for OPG. This difficulty has been experienced by a number of investigators and it is felt that meaningful data will only emerge with a well characterized commercial antibody that is both specific and consistent from batch to batch. At present, such an antibody is not available.

The current study found that metastatic bone stained strongly positive for VEGF and therefore, this ligand could become a simple target for therapy with established agents. No difference was found in VEGF detection between subjects treated with BPs and those who were BP naïve. However, this study included only two patients who were established on BP treatment and hence these findings are questionable. The reason behind such small numbers of BP-treated subjects was the retrospective methodology used in this study. Bone samples were all collected from patients presented to neuroor orthopedic surgeons with spinal cord compression or pathological fractures. The vast majorities of these were new diagnoses of bone metastases and hence were not being actively treated for metastatic bone disease at the time of biopsy. Nevertheless, it is likely that VEGF expression is influenced by BP therapy with work by Santini et al showing a significant decline in plasma VEGF levels among metastatic breast cancer patients after a single treatment with zoledronic acid (42).

Histomorphometry carried out in the current study showed that BP naïve metastatic bone specimens had a slightly increased number of osteoclasts compared to control specimens. This is congruent with the current literature (43). Notably, this study has also demonstrated a complete absence of osteoclasts in BP-treated bone specimens. This is a notable finding, which has not previously been documented in the literature. The majority of histomorphometric data on BP use has been derived from patients with osteoporosis and results have been highly variable. Animal and in vitro studies $(44,45)$ have shown a reduced number of osteoclasts after BP use, although in vivo studies $(46,47)$ have not confirmed this with evidence showing no change in osteoclast number following treatment. It must be stressed that the data derived from this study are based on a very limited number of subjects studied, and therefore, requires further evaluation. While direct antitumor effects of bisphosphonates are scientifically difficult to rule out, the experimental use of OPG in numerous bone metastasis models has provided substantial evidence that turnover suppression is the principal means by which bisphosphonates reduce skeletal tumor burden (40,48-50). The absence of osteoclasts in BP-treated bone metastases is notable; especially as the mechanism for persistent bone resorption in the absence of osteoclasts remains unknown. Animal studies have shown that osteoblast numbers are reduced to $8 \%$ of their baseline number in the later stages of metastatic bone disease. This dramatic loss in osteoblasts likely causes the inability of metastatic bony tissue to remodel even in the presence of BPs (51).

Targeting the RANK/RANKL/OPG pathway is a promising therapeutic option in both postmenopausal osteoporosis $(48,49)$ and in metastatic bone disease $(50)$. A recent placebo controlled trial has shown that a single subcutaneous injection of Denosumab, a fully humanized monoclonal RANKL binding antibody, can dramatically reduce the urinary $\mathrm{N}$-telopeptide/creatinine ratio (uNTx), a validated surrogate marker for bone resorption, in a dosedependent manner (52). This reduction in uNTx was sustained for up to six months. Furthermore, in early phase trials, Denosumab has been shown to decrease uNTx more significantly than IV zoledronic acid in patients with bone metastases (53).

In addition to a significant decline in serum C-telopeptide and uNTx, McClung et al (54) showed an increase in bone mass density after two years of treatment with Denosumab administered every three or six months compared to placebo. Safety profiles of Denosumab in the above studies were all reasonable, with low drop out numbers secondary to adverse events. The results of phase III trials comparing Denusomab to BPs are being awaited and it is hoped that this agent will prove to be a very attractive treatment option to all patients with metastatic bone disease.

In summary, this study has provided in vivo evidence of both the 'seed and soil' and 'vicious circle' theories of dissemination of breast cancer tumor cells to bone. It has been shown that disseminated breast cancer tumor cells appear to express RANK. Moreover, RANKL expressed by osteoblasts in normal bone appears to provide the chemotactic stimulus for propagation of these cells in bone. Subsequently, tumor cells, probably by releasing a variety of cytokines such as $\mathrm{TNF} \alpha$, IL-1 and PTHrP, induce bone stroma in order to produce increasing quantities of RANKL, which in turn stimulates an even greater dissemination of tumor cells. It is also possible that the expression of VEGF by disseminated tumor cells leads to increased differentiation of peripheral blood mononuclear cell into osteoclast-like cells, which are able to resorb bone in an osteoclast-independent manner. These bone resorption pathways warrant further investigation.

This study also showed a dramatic absence of osteoclasts in specimens of patients treated with BPs. This has not been previously reported in the literature. In view of the small sample size, this finding warrants further analysis, if confirmed by larger studies it should lead to increasing attention being paid to the mechanisms of osteoclastindependent bone resorption.

Our group is in the process of carrying out a prospective trial of women with metastatic breast cancer to bone, who have been treated with BPs. These patients will have biopsies of bone and bone marrow and specimens will undergo histo- 
morphometric and immunohistochemical analysis. It is hoped such continued research may not only contribute to the growing body of knowledge of mechanisms of bone resorption and resistance to BPs, but also shed further light on the pathways underlying the development of bone metastases from breast cancer.

\section{References}

1. Ferlay J, Bray F, Pisani P, et al: Globocan 2002: Cancer Incidence, Mortality and Prevalence Worldwide, Version 2.0: IARC CancerBase no. 5, IARC Press, Lyon, 2004.

2. Coleman RE: Skeletal complications of malignancy: Cancer 80: 1588-1594, 1997.

3. Healey JH and Brown HK: Complications of bone metastases. Surgical management. Cancer 88: 2940-2951, 2000.

4. Coleman RE and Rubens RD: The clinical course of bone metastases from breast cancer. Br J Cancer 55: 61-66, 1987.

5. Plunkett TA, Smith P and Rubens RD: Risk of complications from bone metastases in breast cancer implications for management. Eur J Cancer 36: 476-482, 2000

6. Burgess T, Qian YX, Kaufman S, et al: The ligand for osteoprotegerin (OPGL) directly activates mature osteoclasts. J Cell Biol 145: 527-538, 1999

7. Lacey DL, Tan HL, Lu J, et al: Osteoprotegerin ligand modulates murine osteoclast survival in vitro and in vivo. Am J Pathol 157: 435-448, 2000

8. O'Brien EA, Williams J and Marshall MJ: Osteoprotegerin ligand regulates osteoclast adherence to the bone surface in mouse calvaria. Biochem Biophys Res Commun 274: 281-290, 2000.

9. Lacey DL, Timms E, Tan HL, et al: Osteoprotegerin ligand is a cytokine that regulates osteoclast differentiation and activation. Cell 93: 165-176, 1998

10. Schneeweis LA, Willard D and Milla ME: Functional dissociation of osteoprotegerin and its interaction with receptor activator of NF-кB ligand. J Biol Chem 280: 41155-41164, 2005.

11. Yuan YY, Lau AG, Kostenuik PJ, Morony S, Adamu S Asuncion F and Bateman TA: Soluble RANKL has detrimental effects on cortical and trabecular bone volume, mineralization and bone strength in mice. J Bone Miner Res 20: S161-S162, 2005.

12. Yuan YY, Kostenuik PJ, Ominsky MS, et al: Skeletal deterioration induced by RANKL infusion: A model for highturnover bone disease. Osteoporos Int (In press).

13. Yuan YY, Kostenuik PJ, Morony S, Simionescu DT, Basalyga DM and Bateman TA: RANKL infusion as a disease model: Implications for bone and vascular systems. J Bone Miner Res 21: S163, 2006

14. Bucay N, Sarosi I, Dunstan CR, et al: Osteoprotegerin-deficient mice develop early onset osteoporosis and arterial calcification. Genes Dev 12: 1260-1268, 1998

15. Mizuno A, Amizuka N, Irie K, et al: Severe osteoporosis in mice lacking osteoclastogenesis inhibitory factor/osteoprotegerin. Biochem Biophys Res Commun 247: 610-615, 1998.

16. Nakamura M, Udagawa N, Matsuura S, et al: Osteoprotegerin regulates bone formation through a coupling mechanism with bone resorption. Endocrinology 144: 5441-5449, 2003.

17. Kitazawa $\mathrm{S}$ and Kitazawa R: RANK ligand is a prerequisite for cancer-associated osteolytic lesions. J Pathol 198: 228-236, 2002.

18. Thomas RJ, Guise TA, Yin JJ, Elliott J, Horwood NJ, Martin TJ and Gillespie MT: Breast cancer cells interact with osteoblasts to support osteoclast formation. Endocrinology 140: 4451-4458, 1999.

19. Chikatsu N, Takeuchi Y, Tamura Y, et al: Interactions between cancer and bone marrow cells induce osteoclast differentiation factor expression and osteoclast-like cell formation in vitro. Biochem Biophys Res Commun 267: 632-637, 2000.

20. Mancino AT, Klimberg VS, Yamamoto M, Manolagas SC and Abe E: Breast cancer increases osteoclastogenesis by secreting M-CSF and upregulating RANKL in stromal cells. J Surg Res 100: 18-24, 2001.

21. Morony S, Capparelli C, Lee R, Shimamoto G, Boone T, Lacey DL and Dunstan CR: A chimeric form of osteoprotegerin inhibits hypercalcemia and bone resorption induced by IL-1 $\alpha$, TNF- $\alpha$, PTH, PTHrP, and 1,25(OH)2D3. J Bone Miner Res 14 1478-1485, 1999.

22. Fleisch H: Bisphosphonates: mechanism of action. Endocr Rev 9: 80-100, 1998.
23. Coleman RE: Metastatic bone disease: clinical features, pathophysiology and treatment strategies. Cancer Treat Rev 27: 165-176, 2001.

24. Lipton A, Theriault R, Hortobagyi GN, et al: Pamidronate prevents skeletal complications and is effective palliative treatment in women with breast cancer and osteolytic bone metastases - long term follow up of two randomized controlled trials. Cancer 88: 1082-1090, 2000.

25. Galasko CSB: Mechanisms of bone destruction in the development of skeletal metastases. Nature 263: 507-508, 1976.

26. Parfitt AM, Drezner MK, Glorieux FH, et al: Bone histomorphometry: Standardization of nomenclature, symbols, and units. Report of the ASBMR Histomorphometry Nomenclature Committee. J Bone Miner Rel Res 2: 595-610, 1987.

27. Waterman EA, Cross NA, Lippitt JM, et al: The antibody MAB8051 directed against osteoprotegerin detects carbonic anhydrase II: implications for association studies with human cancers. Int J Cancer 121: 1958-1966, 2007.

28. Paget S: The distribution of secondary growths in cancer of the breast. Lancet 1: 571-573, 1889.

29. Orr FW, Varani J, Gondek MD, Ward PA and Mundy GR: Chemotactic responses of tumor cells to products of resorbing bone. Science 203: 176-179, 1979.

30. Kohno N, Aogi K, Minami H, et al: Zoledronic acid significantly reduces skeletal complications compared with placebo in Japanese women with bone metastases from breast cancer: a randomized, placebo-controlled trial. J Clin Oncol 23: 3314-3321, 2005.

31. Hortobagyi GN, Theriault RL, Lipton A, et al: Long-term prevention of skeletal complications of metastatic breast cancer with pamidronate. Protocol 19 Aredia Breast Cancer Study Group. J Clin Oncol 16: 2038-2044, 1998.

32. Theriault RL, Lipton A, Hortobagyi GN, et al: Pamidronate reduces skeletal morbidity in women with advanced breast cancer and lytic bone lesions: a randomized, placebo-controlled trial. Protocol 18 Aredia Breast Cancer Study Group. J Clin Oncol 17: 846-854, 1999.

33. Conte PF, Latreille J, Mauriac L, et al: Delay in progression of bone metastases in breast cancer patients treated with intravenous pamidronate: results from a multinational randomized controlled trial. J Clin Oncol 14: 2552-2559, 1996.

34. Hultborn R, Gundersen S, Ryden S, et al: Efficacy of pamidronate in breast cancer with bone metastases: a randomized, doubleblind placebo-controlled multicenter study. Anticancer Res 19: 3383-3392, 1999.

35. McCloskey E, Guest JF and Kanis J: The clinical and cost considerations of bisphosphonates in preventing bone complications in patients with metastatic breast cancer or multiple myeloma. Drugs 61: 1253-1274, 2001.

36. Mundy GR, Raisz LG, Cooper RA, Schechter GP and Salmon SE: Evidence for the secretion of an osteoclast stimulating factor in myeloma. N Engl J Med 291: 1041-1046, 1974.

37. Cross SS, Harrison RF, Balasubramanian SP, Lippitt JM, Evans CA, Reed MW and Holen I: Expression of osteoprotegerin (OPG), TNF related apoptosis inducing ligand (TRAIL) and receptor activator of nuclear factor KappaB ligand (RANKL) in human breast tumours. J Clin Pathol 59: 56-63, 2006.

38. Mori K, Le Goff B, Charrier C, Battaglia S, Heymann D and Rédini F: DU145 human prostate cancer cells express functional receptor activator of NFKB: New insights in the prostate cancer bone metastasis process. Bone 49: 981-990, 2007.

39. Morrisey C, Kostenuik PJ, Brown LG, Vessella RL and Corey E: Host-derived RANKL is responsible for osteolysis in a C4-2 human prostate cancer xenograft model of experimental bone metastases. BMC Cancer 7: 148, 2007.

40. Kostenuik PJ: Revisiting the seed and soil theory of bone metastasis: New tools, same answer. J Musculoskel Neuron Interact 4: 375-376, 2004.

41. Aldridge SE, Lennard TW, Williams JR and Birch MA: Vascular endothelial growth factor acts as an osteolytic factor in breast cancer metastases to bone. Br J Cancer 92: 1531-1537, 2005.

42. Santini D Vincenzi B, Dicuonzo G, et al: Zoledronic Acid induces significant long lasting modifications of circulating angiogenic factors in cancer patients. Clin Cancer Res 9: 2893-2897, 2003

43. Vukmirovic-Popovic S, Colterjohn N, Lhotak S Duivenvoorden WC, Orr FW and Singh G: Morphological, histomorphometric, and microstructural alterations in human bone metastasis from breast carcinoma. Bone 31: 529-535, 2002. 
44. Pataki A, Müller K, Green JR, Ma YF, Li QN and Jee WS: Effects of short-term treatment with the bisphosphonates zoledronate and pamidronate on rat bone: a comparative histomorphometric study on the cancellous bone formed before, during, and after treatment. Anat Rec 249: 458-468, 1997.

45. Fisher JE, Rogers MJ, Halasy JM, et al: Alendronate mechanism of action: geranylgeraniol, an intermediate in the mevalonate pathway, prevents inhibition of osteoclast formation, bone resorption, and kinase activation in vitro. Proc Natl Acad Sci USA 96: 133-138, 1999

46. Chavassieux PM, Arlot ME, Reda C, Wel L, Yates AJ and Meunier PJ: Histomorphometric assessment of the long-term effects of alendronate on bone quality and remodeling in patients with osteoporosis. J Clin Invest 100: 1475-1480, 1997.

47. Bravenboer N, Papapoulos SE, Holzmann P, Hamdy NAT, Netelenbos JC and Lips P: Bone histomorphometric evaluation of pamidronate treatment in clinically manifest osteoporosis. Osteoporos Int 9: 489-493, 1999.

48. Zheng Y, Zhou H, Brennan K, Blair JM, Modzelewski J, Seibel MJ and Dunstan CR: Inhibition of bone resorption, rather than direct cytotoxicity, mediates the anti-tumor actions of ibandronate and osteoprotegerin in a murine model of breast cancer bone metastasis. Bone 40: 471-478, 2007.

49. Quinn JE, Brown LG, Zhang J, Keller ET, Vessella RL and Corey E: Comparison of Fc-osteoprotegerin and zoledronic acid activities suggest that zoledronic acid inhibits prostate cancer in bone by indirect mechanisms. Prostate Cancer Prostatic Dis 8: 253-259, 2005
50. Yaccoby S, Pearse RN, Johnson CL, Barlogie B, Choi Y and Epstein J: Myeloma interacts with the bone marrow microenvironment to induce osteoclastogenesis and is depenendent on osteoclast activity. Br J Haematol 116: 278-290, 2002.

51. Phadke PA, Mercer RR, Harms JF, et al: Kinetics of metastatic breast cancer cell trafficking in bone. Clin Cancer Res 12: 1431-1440, 2006.

52. Bekker PJ: A single-dose placebo-controlled study of AMG 162 , a fully human monoclonal antibody to RANKL, in postmenopausal women. J Bone Miner Rel 29: 2274-2281, 2005.

53. Suarez T, Fizazi K, Rahim Y, Wilson J, Fan M, Jun S and Lipton A: A randomized trial of denosumab (AMG 162) versus intravenous (IV) bisphosphonates (BP) in cancer patients (pts) with bone metastases (BM) on established IV BP and evidence of elevated bone resorption. J Clin Oncol ASCO Annual Meeting Proc Part I 24: Abs 8562, 2006.

54. McClung MR, Lewiecki EM, Cohen SB, et al: Denosumab in postmenopausal women with low bone mineral density. N Engl J Med 354: 821-831, 2006. 